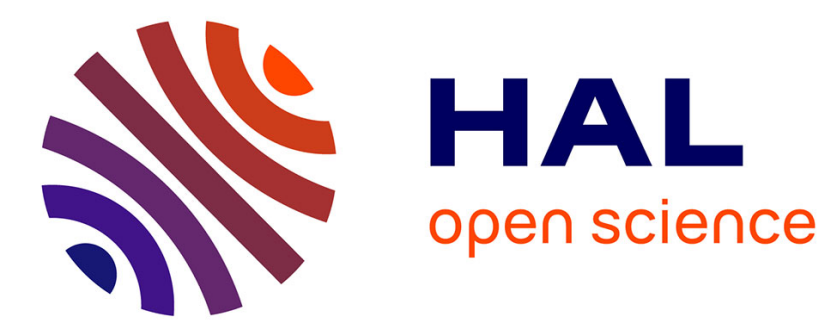

\title{
Sauvegarde du patrimoine sonore
}

\author{
J.-M. Fontaine
}

\section{To cite this version:}

J.-M. Fontaine. Sauvegarde du patrimoine sonore. Journal de Physique IV Proceedings, 1994, 04

(C5), pp.C7-241-C7-244. 10.1051/jp4:1994548 . jpa-00253043

\section{HAL Id: jpa-00253043 https://hal.science/jpa-00253043}

Submitted on 1 Jan 1994

HAL is a multi-disciplinary open access archive for the deposit and dissemination of scientific research documents, whether they are published or not. The documents may come from teaching and research institutions in France or abroad, or from public or private research centers.
L'archive ouverte pluridisciplinaire HAL, est destinée au dépôt et à la diffusion de documents scientifiques de niveau recherche, publiés ou non, émanant des établissements d'enseignement et de recherche français ou étrangers, des laboratoires publics ou privés. 


\section{Sauvegarde du patrimoine sonore}

\section{J.-M. FONTAINE}

Ministère de la Culture, Paris VI, URA 879 du CNRS, Laboratoire de Mécanique Physique, Le Val Joyeux CD 11, 78450 Villepreux, France

Il n'est pas de mesures universelles de préservation de collections sonores d'importance patrimoniale constituées des témoins de l'histoire de l'enregistrement : cylindres, disques noirs, fils et bandes magnétiques, disques optiques, ... Les actions de sauvegarde des collections d'enregistrement sonores sont extrêmement variées et bien ciblées, surtout lorsque les techniques analogiques sont concernées. Peu-à-peu, les modes numériques d'enregistrement modifient le concept même de conservation des supports au profit de la considération de la seule information que l'on doit faire passer d'un système à un autre.

There is no universal measurement of preservation of patrimonial sound collections. These collections are often composed of historical witness recordings : cylinders, black records, magnetic wires and tapes, optical discs, ... Actions that have been performed to protect audio record collections are extremely varied and specific, especially when analogical technology is concerned. But digital recording processes are now modifying the preservation concept of carriers which consist of dealing the only information that has to be brought from one system to another.

La conservation des enregistrements sonores est perçue de manière bien différente par le particulier tout autant attaché au support qu'au contenu lui même (le "collectionneur"), les créateurs, la profession éditoriale qui réédite périodiquement son stock, les laboratoires de recherche sur le son. Pour la Bibliothèque Nationale - Département de la Phonothèque chargée de préserver des collections d'intérêt patrimonial, aucune limitation thématique des fonds, aucune limitation de durée de conservation ne sont fixées. Notre contribution s'effectue dans le cadre d'une Convention passée entre la Bibliothèque Nationale, Paris 6 et le CNRS.

Il faut assurer la permanence de linformation et la pérennité du matériel permettant l'accès à celle-ci. La première difficulté, pour les collections d'enregistrements non édités notamment (celles que détiennent bon nombre de chercheurs et de laboratoires d'acoustique), provient de linsuffisance des données descriptives du contenu des enregistrements. Les informations entourant la constitution des enregistrements deviennent cruciales lorsque l'on quitte le contexte expérimental original. 


\section{LA MODELISATION DU VIEILLISSEMENT ET SES LIMITES.}

L'interprétation théorique de certains phénomènes liés au vieillissement a été modélisée avec succès : l'effet de copie dans le domaine du magnétisme, les processus d'évolution chimique par hydrolyse du liant polyuréthane des bandes magnétiques, etc. Mais plus généralement, le vieillissement naturel voit l'intrication de mécanismes chimiques élémentaires qui doivent être explicités. Il faut en outre rappeler que l'évolution des propriétés physiques n'est pas toujours une relation simple de la dégradation chimique. Malgré ces difficultés, l'établissement de la relation entre les altérations physico-chimiques et la perte d'informations "inscrites" sur le support devra être tentée car nous sommes confrontés essentiellement à la dégradation du signal. La modélisation de l'évolution du taux d'erreur des disques à lecture optique a été ajustée à la loi d'Arrhénius généralisée sans que les processus de dégradation physico-chimique pouvant engendrer une telle évolution n'aient été établis à ce jour. Aussi convient-il de rester très prudent sur les estimations de comportement qui pourraient être tentées par extrapolation. Pour étayer cette remarque, nous considérons deux disques, choisis au hasard de la production, $\mathrm{CD} 1$ et $\mathrm{CD} 2$ soumis à des traitements de vieillissement thermique identiques. Le taux d'erreurs blocs (BLER), qui constitue le paramètre de premier rang pour mesurer l'état de l'information sur le disque, augmente à mesure que l'exposition à $80^{\circ} \mathrm{C}(\mathrm{sec})$ se prolonge. Mais cette évolution présente d'importantes différences selon le rayon : les défauts deviennent beaucoup plus importants sur la partie centrale du disque CD1. Le disque CD2 présente quant à lui une tendance tout à fait contraire. L'analyse du profil de l'évolution chimique de la couche du vernis avant essais (microspectrophotométie IRTF par réflexion, Laboratoire Centre National d'Evaluation de Photoprotection, Université Blaise Pascal / Clermont II) a montré que de telles variations ne pouvaient être interprétées simplement à partir de variation d'épaisseur. L'analyse du vernis vieilli fait apparaître des évolutions chimiques plus marquées dans les zones concernées. Dans cette première phase d'investigation, l'observation de l'évolution de l'ensemble des spectres a permis d'établir que la thermooxydation du vernis pouvait être ainsi reliée à l'apparition des taux d'erreur.
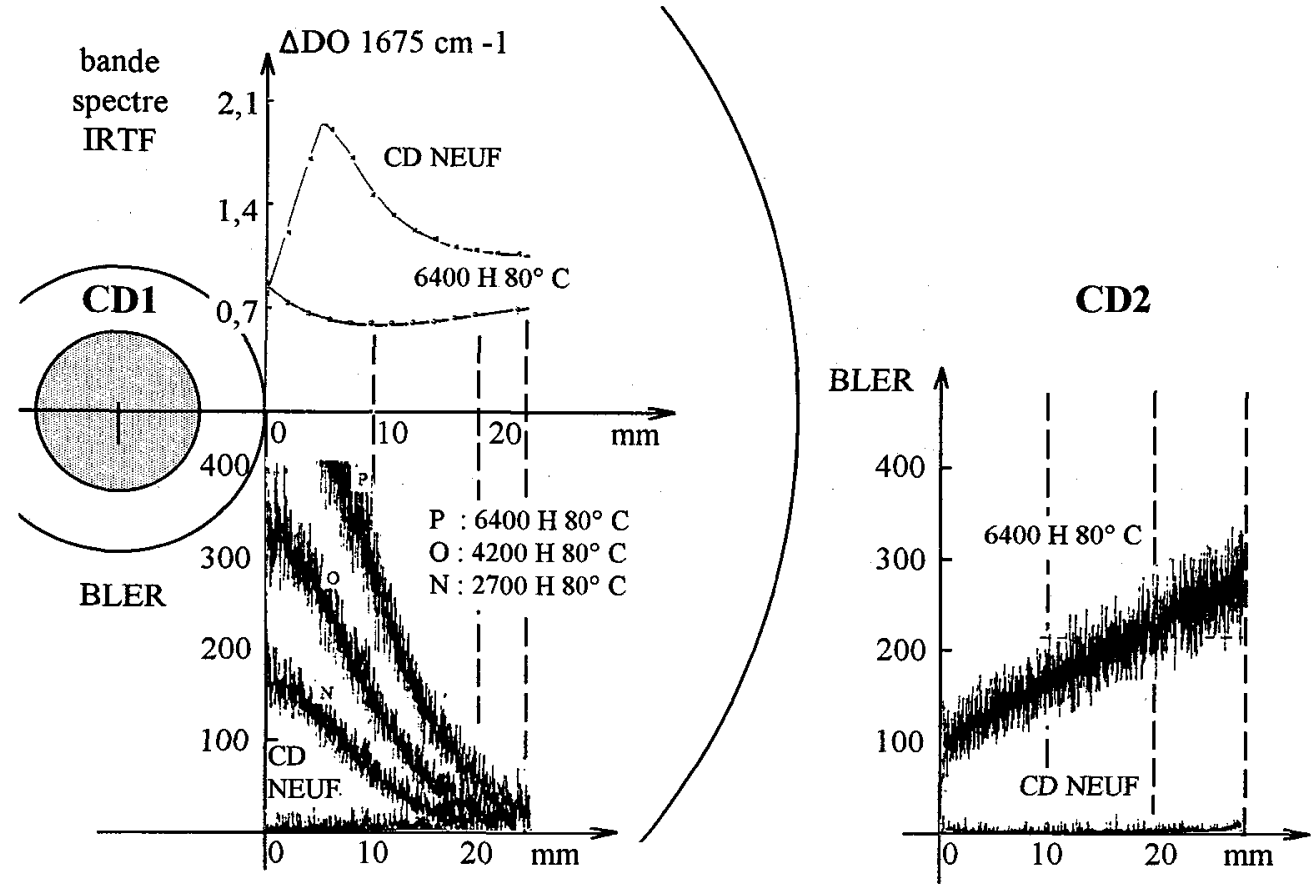


\section{LES TERRAINS D'ACTIONS}

Les terrains d'actions concernant la gestion et la communication de collections d'enregistrements sonores sont nombreux : protection des documents originaux (décontamination d'attaques fongiques, nettoyage, restaurations de bandes inondées, ...), définition et mise en oeuvre des conditions de stockage adaptées aux différents supports (conditions climatiques, mesures préventives contre l'incendie, ...), maintenance des systèmes de lecture, restauration des signaux dégradés, transfert de l'enregistrement vers des supports de substitution fiables. Sans oublier le développement des outils bibliothéconomiques et les moyens de communication sans lesquels la conservation dépourvue de moyens de diffusion ne saurait avoir de sens ...

Ces dernières années, les avancées les plus marquantes se sont manifestées dans les domaines suivants :

2.1 Les études de vieillissement chimique qui permettent de mieux comprendre les processus de dégradation des supports, de prendre les dispositions de stockage les plus appropriées et de supputer les durées de vie

2.2 Les moyens de lecture des supports dits mécaniques (cylindres et disques noirs) :

2.2.1. Dispositifs de lecture optique avec fibre légère (Ecole Polytechnique Fédérale de Lausanne) ou à faisceau laser sans contact (Platine Finial / ELP Co). De tels procédés optiques offrent une chance supplémentaire de lire les documents particulièrement dégradés, ils facilitent le repérage des plages; mais encore, ils peuvent se montrer moins réducteurs que la pointe du stylet vis-à-vis de la quantité l'information contenue dans les sillons. Fort heureusement, pour les applications courantes, il subsiste (mais pour combien de temps ?) des fabrications de platines, de bras et de cellules de haute qualité correspondant aux exigences des disques de confections et d'usures très variables.

2.2.2. Préamplificateurs autorisant une très large palette de courbes caractéristiques de lecture que l'on choisit sur des critères historiques et esthétiques. Placés devant le manque de préamplis disponibles sur le marché, nous avons été amenés à réaliser, avec la société M.E.R. différents modèles spécifiques pour nos applications.

2.3 Le développement d'outils de traitements de signal permettant de mieux évaluer la nature des défauts de qualité du son (localiser l'origine des dégradations, classifier celles-ci, choisir telle ou telle thérapeutique, ...) et d'agir avec une efficacité accrue sur les différents types de signaux perturbateurs, sur des messages devenus diaphanes, ...

Nous travaillons (cf. communication de M. ZENG HONG) dans ce sens en collaboration avec l' Université du Maine qui a acquis une expérience certaine sur ces questions.

Bien évidemment, l'emploi de procédés risquant de modifier l'essence même d'une information lorsqu'elle est de référence - ne doit pas s'opérer sans respecter les principes fondamentaux de protection de l'intégrité de celle-ci. Lés studios sont organisés de telle manière que les opérations de transfert de conservation qui ne comportent strictement aucun traitement soient effectuées parallèlement à un transfert dit de communication (consommation) qui peut bénéficier très largement des techniques de traitement du signal d'une époque donnée.

2.4 Les procédures de transfert des enregistrements assistés par ordinateur : indexation des plages (sur la base de l'enregistrement du Time Code), identification des supports numériques (User Bits). A la description du programme sont jointes les informations recueillies pendant les opérations sur l'état des documents, les conditions de lecture et la qualité de l'enregistrement 
obtenue. La liaison est effectuée avec la base de données discographiques de la Bibliothèque Nationale (OPALINE). Ce logiciel permet le dialogue avec trois enregistreurs cibles possibles (magnétophone analogique, numérique, $\mathrm{CD}$ enregistrable éventuellement, etc. ) a été développé sous WINDOWS et mis en oeuvre dans les studios de la Phonothèque où des transferts massifs de disques noirs sont en cours pour répondre au double objectif d'assurer la conservation (par dédoublement) des enregistrements et de les mettre à la disposition du public de la Bibliothèque Nationale de France.

2.5 La réalisation de supports spécifiques destinés à être conservés sur de très longues périodes (disque de verre gravé à lecture optique de Digipress, utilisation de poudres ferromagnétiques stables pour les bandes numériques, etc.)

\section{UNE STRATEGIE DE REPORTS}

Contrairement à la proposition de supports "durcis", il est à noter l'absence de propositions spécifiques concernant un élément majeur de l'enregistrement numérique du son : le Format. La conservation des enregistrements analogiques pouvait pratiquement être identifiée à celle de son support. La conservation de l'enregistrement numérique ne se conçoit, à l'évidence qu'à travers le système qui permet d'accéder à l'information contenue sur un support qui lui est propre, physiquement et par son protocole d'inscription. Naturellement, pour un disque aussi répandu que le Disque Compact Audio (la Phonothèque détient - fin 1993 - quelques 60000 disques), nous nous préoccupons de la survie matérielle du support. Mais pour l'ensemble des documents numériques, la question du report des données sur un système relais se pose sur la base de critères de qualité, de capacité, d'harmonisation des format d'écriture et des supports, de fiabilité, de disponibilité d'accès pendant une longue durée, et déjà des modalités des futurs transferts en masse.

Les techniques de communication de linformation bénéficient de moyens puissants de réduction de débit, mais il faut bien souligner que la compression avec élimination des informations décrétées inutiles n'est pas applicable dans le cadre de transfert de données destinées à être conservées. Les techniques de compactage ne rencontrent pas de telles restrictions si toutefois elles n'augmentent pas de manière significative la sensibilité de l'enregistrement aux altérations.

S'il n'existe pas de système répondant à l'ensemble de ces critères, certains formats numériques de grande capacité offrent certains avantages : DIR-1000 de Sony (dérivé du format numérique I D1), Nagra D de Kudelski, etc. Le disque de verre gravé, les disques enregistrables (CD-R, disques WORM) constituent aussi une proposition de report judicieuse dans certains cas (systèmes "juke-box").

D'autres réalisations effectuées dans des secteurs différents du notre laissent espérer des transferts de technologie profitables pour les collections sonores (et vidéographiques) de très grande capacité.

Il nous faut accepter l'idée de non permanence de l'information fixée sous forme numérique sur un support / système donné et étudier la mise en place d'une stratégie de reports sur systèmes relais espacés d'une durée difficile à estimer. Ces transferts, placés dans une logique de reports récursifs continus, doivent être maîtrisés sur un plan technique et se montrer financièrement supportables : le risque de perte des informations devient considérable si une interruption supérieure à la durée de vie d'un système d'accès - maintenance comprise - devait intervenir, notamment pour des raisons financières. 